\title{
Ipercortisolismo funzionale e sue possibili conseguenze cliniche
}

\author{
Marianna Martino ${ }^{1} \cdot$ Giorgio Arnaldi $^{1}$
}

Accettato: 9 dicembre 2020 / Pubblicato online: 31 maggio 2021

(c) The Author(s) 2021

Sommario L'ipercortisolismo funzionale rappresenta una condizione di attivazione cronica dell' asse ipotalamo-ipofisisurrene che si verifica in condizioni cliniche eterogenee (depressione e altri disordini psichici, disturbi del comportamento alimentare, diabete mellito, obesità, alcolismo, sindrome dell'ovaio policistico, sindrome delle apnee ostruttive notturne, lavoro a turni). Solitamente è un ipercortisolismo di lieve entità e reversibile alla remissione della condizione sottostante. Deve essere distinto dalla Sindrome di Cushing con la quale, però, condivide manifestazioni cliniche, alterazioni biochimiche e problematiche di diagnostica differenziale. Si può ipotizzare che l'ipercortisolismo funzionale abbia comunque un' azione deleteria tessuto-specifica. In questa rassegna verranno illustrati sinteticamente meccanismi ed effetti nocivi dell'ipercortisolismo funzionale.

Parole chiave Ipercortisolismo funzionale . PseudoCushing · Depressione maggiore - Disturbi comportamento alimentare $\cdot$ Alcoolismo $\cdot$ PCOS

\section{Introduzione}

Si definisce ipercortisolismo funzionale (FH) l'attivazione cronica dell'asse ipotalamo-ipofisi-surrene (HPA), solitamente di entità lieve e reversibile alla remissione della

Proposto da Carla Scaroni.

Informazioni Supplementari La versione online contiene materiale supplementare disponibile su

https://doi.org/10.1007/s40619-021-00865-w.

$凶$ G. Arnaldi

g.arnaldi@staff.univpm.it

1 Clinica di Endocrinologia e Malattie del Metabolismo, A.O.U. Ospedali Riuniti di Ancona, Università Politecnica delle Marche, Ancona, Italia condizione sottostante, che si verifica in corso di depressione maggiore e altri disturbi psichici, disturbi del comportamento alimentare (DCA), diabete mellito, obesità essenziale, alcolismo, sindrome dell'ovaio policistico, sindrome delle apnee ostruttive notturne, lavoro a turni. Sebbene sostenuto da meccanismi fisiopatologici disturbo-specifici, l'FH può essere indistinguibile dalla Sindrome di Cushing (CS), con la quale condivide manifestazioni cliniche (Fig. 1), alterazioni biochimiche e problematiche di diagnostica differenziale, tanto da esser noto anche col nome di pseudoCushing $[1,2]$. Similmente a quanto accade nella CS, si ipotizza che sia l'azione deleteria tessuto-specifica del cortisolo la responsabile delle complicanze sistemiche (principalmente cardiovascolari e metaboliche) e dell'eccesso di mortalità osservabile anche nelle varie situazioni di FH [3]. In questa rassegna verranno illustrati sinteticamente meccanismi ed effetti nocivi dell'FH nelle principali condizioni che lo sottendono.

\section{Depressione maggiore e altri disturbi psichici}

Fino al $30 \%$ dei pazienti affetti da depressione maggiore (MD), prevalentemente di genere maschile, sviluppa FH [2].

L'iperattivazione del sistema limbico stimola la produzione di CRH che si autoamplifica a causa della desensitizzazione e down-regulation dei recettori per i glucocorticoidi situati nell'ippocampo dove, peraltro, citochine infiammatorie inducono modifiche neuroanatomiche e funzionali [1]. $\mathrm{Ne}$ risultano un'alterata pulsatilità dell'ormone adrenocorticotropo (ACTH) e un sovvertimento del ritmo circadiano del cortisolo (picchi mattutini più accentuati e mancato nadir serale), con riscontro morfologico di iperplasia surrenalica reversibile $[1,2]$. Concomita una maggior biodisponibilità tissutale del cortisolo per ridotta attività degli enzimi 


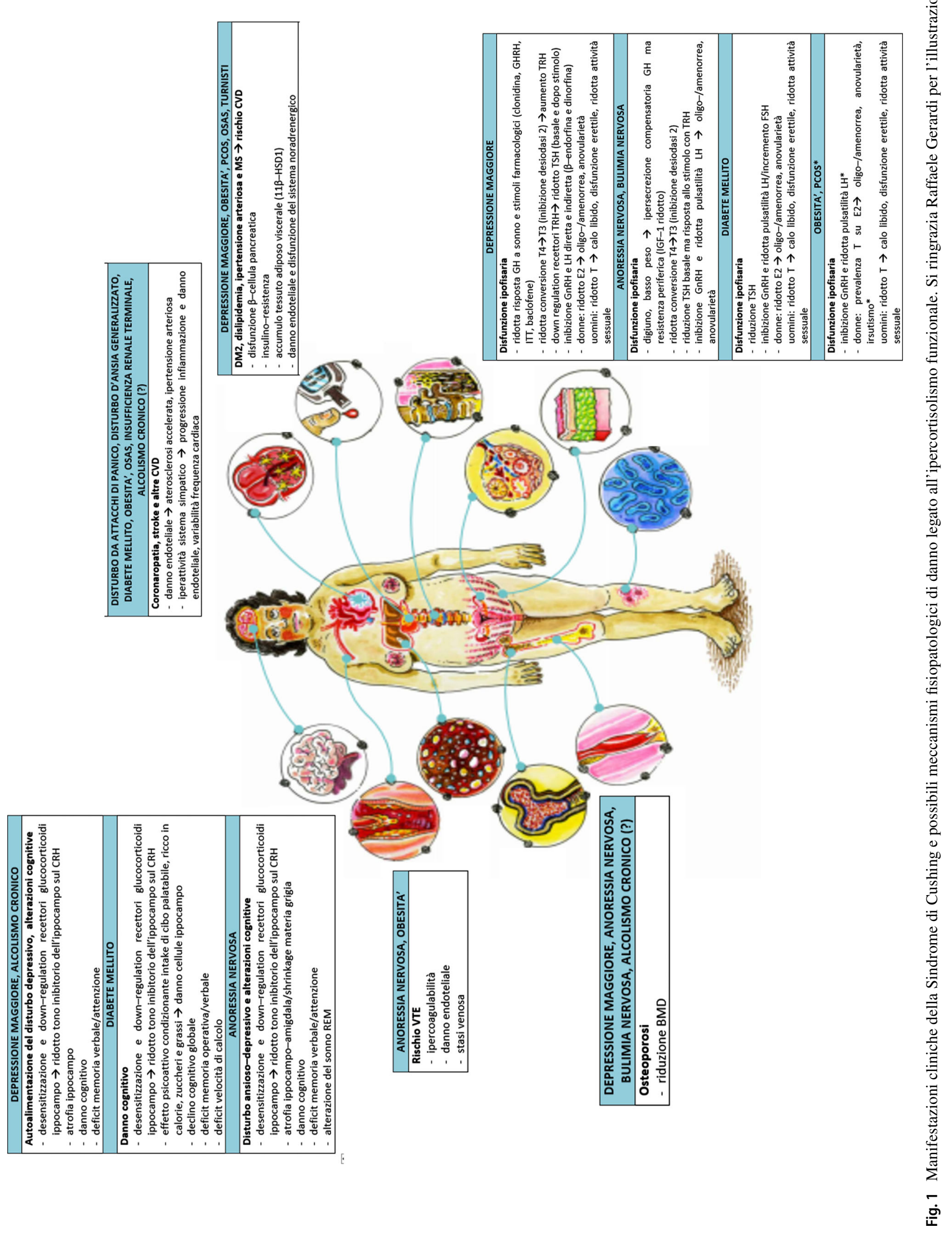


$5 \alpha$-reduttasi tipo 1 e $11 \beta$-idrossisteroidodeidrogenasi tipo 2 (11 $\beta$-HSD2), deputati alla sua inattivazione intracellulare: tale alterazione può persistere poiché alcuni psicofarmaci inducono modifiche solo parziali nell'attività enzimatica [2].

Probabilmente l'FH è responsabile di molte complicanze sistemiche della MD (Fig. 1), sindrome metabolica (MS) e mortalità cardiovascolare (CV) in primis [1]. Eccessivi livelli di cortisolo potrebbero avere un'azione diretta sulla $\beta$-cellula pancreatica, causandone disfunzione [1]. L'insorgenza di MD e delle altre componenti della MS sembra, inoltre, favorita dall'aumento della quota di tessuto adiposo intra-addominale tipico della MD e verosimilmente ipercortisolismo-indotto: Thakore e collaboratori hanno infatti evidenziato una correlazione positiva tra cortisolo plasmatico e quota di adipe viscerale misurato alla tomografia computerizzata in 7 donne con MD [4]. Anche la disfunzione endoteliale responsabile dell' aumentato rischio $\mathrm{CV}$ nella MD sembra mediata dal cortisolo, come testimoniato dal miglioramento significativo della dilatazione flussomediata dell'arteria brachiale di soggetti con MD dopo 6 ore dalla somministrazione di metirapone rispetto a placebo [5]. Come precedentemente accennato, l'FH è in grado di peggiorare il quadro di MD sostenendola dal punto di vista patogenetico: ciò è stato confermato da numerosi studi clinici che hanno riportato un miglioramento dei sintomi di MD con l'utilizzo di inibitori della steroidogenesi surrenalica, antagonisti recettoriali dei glucocorticoidi e antagonisti del recettore del CRH tipo 1 (CRF1) [6]. L'azione nociva del cortisolo si esplica a livello dell'ippocampo, struttura critica per l'apprendimento e la memoria a lungo termine che, fisiologicamente, esprime ad alta densità recettori per i glucocorticoidi [1]. In presenza di FH l'espressione di tali recettori viene down-regolata, con conseguente riduzione dell'inibizione dell'ippocampo sulla secrezione ipotalamica di CRH e autoalimentazione dell'FH stesso. Nei pazienti con MD psicotica, il danno si manifesta nell'ambito cognitivo e della memoria verbale, è biochimicamente correlato a un alterato ritmo circadiano del cortisolo e morfologicamente all'atrofia dell'ippocampo [1].

Sebbene ancora da chiarire in via definitiva, l'FH potrebbe giocare un ruolo chiave nello sviluppo di osteoporosi [1]. Livelli di cortisolo urinario e plasmatico più elevati in donne depresse sono risultati correlati in maniera negativa con la mineralizzazione ossea (BMD), peraltro ridotta in più siti ossei [7]. Inoltre, la secrezione di ormone somatotropo $(\mathrm{GH})$ in risposta al sonno e a differenti stimoli farmacologici sembra ridotta [1]; l'eccesso di cortisolo inibisce inoltre l'enzima desiodasi-2, con conseguente riduzione della conversione da tiroxina (T4) a triiodotironina (T3) e, attraverso una down-regulation dei recettori del TSH releasing hormo$n e$ (TRH) ipotalamico, della secrezione di ormone tireostimolante (TSH) sia in condizioni basali che dopo stimolo con
TRH; infine, l'ipersecrezione di CRH è associata a riduzione nella secrezione di ormone di rilascio delle gonadotropine $(\mathrm{GnRH})$ e ormone luteinizzante $(\mathrm{LH})$, con alterazioni del ciclo mestruale, calo della libido e disfunzione erettile negli uomini: per questi ultimi è stata anche dimostrata una ripresa della funzione gonadica in risposta alla terapia psicofarmacologica, in parallelo con la riduzione dei livelli di cortisolo $[1,8]$.

Anche nei pazienti con disturbo da attacchi di panico (PD) o disturbo d'ansia generalizzato (GAD) è stata riportata la presenza di un FH di grado proporzionale alla severità dei sintomi (comportamento agorafobico, ansia/depressione e loro associazione agli attacchi di panico): tuttavia, i risultati degli studi sono contrastanti e non vi sono ancora dati dirimenti in merito al ruolo, causale o consequenziale, che l'eccesso di cortisolo riveste nei confronti di tali disturbi [1]. La disciplina emergente della cardiologia comportamentale considera l'FH una risposta centrale evocata da ansia e depressione e ne enfatizza il ruolo di fattore promuovente la patologia coronarica e cardiovascolare che molto frequentemente interessa i pazienti psichiatrici [9].

\section{Disturbi del comportamento alimentare}

Nei pazienti con DCA (anoressia nervosa, AN, e bulimia nervosa, $\mathrm{BN}$ ) è stata ampiamente documentata un'iperattivazione dell' asse HPA [2].

In questo caso, allo sviluppo dell'FH concorrono una ridotta clearance del cortisolo, un'alterazione nella sua affinità di legame alla Cortisol Binding Globulin (CBG), una resistenza recettoriale e una spiccata produzione di CRH: è a questi ultimi due elementi, in particolare al potente effetto anoressizzante del $\mathrm{CRH}$, peraltro tipicamente associato a normali livelli di ACTH e ritmo circadiano del cortisolo conservato, che può essere ascritta almeno in parte l'assenza del classico fenotipo cushingoide $[1,2]$.

Se da un lato l'FH rappresenta un meccanismo adattativo allo stress digiuno-indotto (AN) o la risposta agli stimoli che fisiologicamente derivano dall'ingestione di pasti eccessivi (BN), dall'altro lato la dimostrazione di una persistente iperattivazione dell'asse HPA, anche dopo remissione, ne suggerisce un contributo di tipo patogenetico e a sostegno di questa ipotesi vi è il riscontro di una preferenziale distribuzione centripeta del tessuto adiposo nelle pazienti con AN in remissione [2].

L'FH determinerebbe importanti conseguenze sistemiche (Fig. 1). In particolare, è stata documentata un' associazione tra livelli di cortisolo e presenza/severità di ansia e depressione, nonché insorgenza di deficit dell'attenzione e cognitivi nelle pazienti con AN [1]: in uno studio di risonanza magnetica voxel-based morphometry (VBM) su 12 adolescenti con AN, tali manifestazioni sembrerebbero ascrivibili allo shrinkage della materia grigia delle aree temporali e 
parietali, direttamente indotto dal cortisolo e reversibile in maniera fortemente correlata alla riduzione dell'ormone dopo trattamento nutrizionale in reparti dedicati [10]. L'effetto deleterio del cortisolo si somma poi alle carenze nutrizionali e all'ipogonadismo funzionale che caratterizza i disturbi del comportamento alimentare contribuendo alla riduzione della BMD tipica delle pazienti con AN, dove l'ipercortisolismo è stato considerato uno tra i migliori biomarker per valutare il rischio di osteopenia [11], ma osservabile anche in alcuni casi di BN dove, tuttavia, solo uno studio ha rilevato correlazioni negative tra cortisolo plasmatico e BMD total body [12]. Anche nei DCA si confermano le modifiche adattative a carico degli assi gonadotropo (alterazione nella secrezione del GnRH con compromissione della pulsatilità dell'LH risultante in oligo-/amenorrea e anovularietà anche nelle pazienti con $\mathrm{BN}$ e normale indice di massa corporea (BMI) e tireotropo (inibizione della desiodasi-2 con shift da $\mathrm{T} 4$ a reverse $\mathrm{T} 3$, riduzione del TSH basale ma risposta allo stimolo con TRH) e si evidenzia, nel contesto di un'iperattivazione compensatoria dell'asse somatotropo, una resistenza acquisita al GH dovuta a basso peso e digiuno, con livelli di Insulin-like Growth Factor-1 (IGF-1) inversamente correlati a quelli del cortisolo [1]. Infine, un recente studio danese ha posto l'accento sul possibile ruolo predisponente dell'FH nei confronti del tromboembolismo venoso (VTE) che può verificarsi durante il refeeding delle pazienti con AN: aggiungendo danno endoteliale e ipercoagulabilità alla stasi venosa da ritenzione idrica e alterata permeabilità vascolare che si osserva nelle fasi precoci della re-nutrizione, l'FH completerebbe la triade di Virchow alla base del VTE [13].

\section{Alcolismo}

L'alcolismo è uno degli stati di pseudoCushing meglio conosciuti, potendo mimare sia i segni obiettivi (facies lunaris, miopatia, astenia, obesità tronculare, ipertensione arteriosa e striae rubrae), sia le alterazioni biochimiche tipici della CS, soprattutto nelle donne [1,2].

In questo caso l'FH, reversibile dopo alcune settimane di recupero dal potus, è sostenuto da meccanismi centrali e periferici: abuso e astinenza da alcool, infatti, stimolano la produzione di CRH sia direttamente che attraverso l'inibizione del feedback ippocampale e a ciò si somma la ridotta clearance del cortisolo da induzione dell'enzima $11 \beta$-HSD1 secondaria all'epatopatia alcolica $[1,2]$.

È stato ampiamente dimostrato che l'FH è in grado di favorire l'insorgenza e aggravare la severità e l'autopercezione di depressione e alterazioni cognitive non solo nell'alcolismo cronico, ma anche nei pazienti in astinenza e molto tempo dopo l'avvenuta sospensione [1]. Sulla scorta di queste evidenze è stato recentemente ipotizzato l'uti- lizzo del Mifepristone, antagonista recettoriale dei glucocorticoidi, nel trattamento della depressione e del deficit di attenzione nella fase di disintossicazione da alcool [14]: purtroppo, questo trial clinico in doppio cieco randomizzato placebo-controllato non ha fornito risultati conclusivi in merito.

Sorprendentemente, non ci sono evidenze sufficienti a chiarire definitivamente la relazione tra $\mathrm{FH}$ e ipertensione arteriosa, riscontrata in circa il $50 \%$ dei pazienti alcolisti e associata a elevati livelli di cortisolo non solo in termini di valori pressori sistolici ma anche di variabilità [1]; similmente, mancano dati a sostegno di un ruolo dell'FH nella ben nota associazione tra alcolismo e ridotta BMD [1].

\section{Obesità essenziale}

Negli ultimi anni crescenti evidenze hanno posto l'accento sul ruolo del cortisolo nella patogenesi dell'obesità, condizione pandemica a rapida crescita e con conseguenze importanti per la salute pubblica [15]: il cortisolo, infatti, induce una redistribuzione del tessuto adiposo bianco in senso centripeto, stimola l'appetito favorendo l'intake preferenziale di cibi altamente calorici e, come evidente nei pazienti con CS, si associa allo sviluppo di MS e malattie CV [15].

Nella società moderna, la pandemia di obesità ha coinciso con un importante aumento dei fattori che promuovono la sintesi di cortisolo, quali lo stress cronico, il consumo di cibi ad alto indice glicemico e la riduzione del sonno, suggerendo quindi l'esistenza di un circolo vizioso dove cortisolo, obesità e stress interagiscono autoalimentandosi a vicenda $[1,15]$. A supporto di questa ipotesi vi è l'evidenza di un aumento del 9,8\% nei livelli di cortisolo nei capelli ogni $+2,5$ punti di BMI [16] e, in uno studio su oltre 3.000 bambini di 6 anni, di un rischio 10 volte superiore di obesità addominale in coloro che avevano i livelli di cortisolo più alti [17].

Negli individui obesi la secrezione di cortisolo in risposta a stressors è spesso elevata, ma le concentrazioni circolanti sono normali/basse, probabilmente a causa di cambiamenti tessuto-specifici nel metabolismo dell'ormone stesso (esso è iperprodotto nel tessuto adiposo, ma l'aumentata attività della $5 \alpha$-reduttasi tipo 1 lo inattiva a livello epatico e rende ragione dell'iperattivazione compensatoria dell' asse HPA con aumento di cortisolo libero urinario e spiccata risposta ai test di stimolo); inoltre, la sensibilità ipofisaria al feedback negativo esercitato dai glucocorticoidi è preservata [2].

L'obesità può presentarsi con la tipica distribuzione centripeta in rapporto alla maggior densità di recettori dei glucocorticoidi nell' adipe viscerale rispetto a quello sottocutaneo e all'aumentata espressione da parte del tessuto adiposo dell' $11 \beta$-HSD1, enzima microsomiale che ha la funzione di rigenerare a livello locale cortisolo attivo da cortisone inattivo, regolandone l'accesso ai recettori intracellulari e pro- 
muovendo differenziazione dei pre-adipociti e ipertrofia degli adipociti $[2,15]$; a ciò si sommano la variabile sensibilità individuale all'azione dei glucocorticoidi e l'eventuale presenza di fattori ambientali e comportamentali predisponenti l'accumulo di adipe addominale [15].

La principale conseguenza clinica potenzialmente correlabile all'FH è lo sviluppo di malattie CV [18]: delle varie forme di obesità quella addominale, identificata dall' aumento della circonferenza vita e - nelle donne - correlata ai livelli di cortisolo in termini di BMI e tessuto adiposo viscerale, presenta la più stretta associazione con MS, insulinoresistenza (HOMA index), dislipidemia aterogenica, stato proinfiammatorio, protrombotico e coronaropatia [18].

Anche l'asse gonadotropo risente dell'FH, ma con alcune differenze di genere [1]. Se, infatti, l'iperattivazione dell'asse HPA ha un effetto inibitorio sugli ormoni sessuali in entrambi i generi, gli effetti clinici che ne risultano sono diversi, con una soppressione del testosterone che conduce all' $i$ pogonadismo maschile e un'eccessiva produzione di testosterone nelle donne che causa irregolarità mestruali e un fenotipo simile a quello della sindrome dell'ovaio policistico [1].

\section{Diabete mellito (DM)}

La prevalenza di una CS nei pazienti con DM è compresa tra 0,6 e 9,4\%, ragion per cui uno screening per l'ipercortisolismo è giustificato in questo contesto, specialmente nei pazienti con iperglicemia incontrollata nonostante terapia ottimale $[1,3]$.

In questo caso va comunque considerata la possibilità di trovarsi di fronte a un FH, in cui l'iperattivazione dell'asse HPA secondaria agli effetti dello scarso controllo glicemico sull'ippocampo si associa all' aumentata espressione e attività della $11 \beta$-HSD1 sottocutanea e viscerale tipica degli stati di MS e, come emerge dallo studio DIALECT-1, del DM complicato da danno renale [1, 19]: tale enzima potrebbe giocare un ruolo chiave nel favorire le complicanze cardiovascolari del DM (cardiopatia ischemica, aterosclerosi carotidea, nefropatia, retinopatia e neuropatia) anche in assenza di franche alterazioni nei livelli di cortisolo circolante [1].

$\mathrm{Se}$, infatti, da un lato l'esistenza di correlazioni positive tra cortisolo e glicemia a digiuno, emoglobina glicata, $\mathrm{Ho}$ meostasis Model Assessment (HOMA) index è accertata nei pazienti con DM 1 e DM 2 ipercortisolemici, essa dall'altro è stata confermata anche in un'ampia coorte di pazienti con DM2 normocortisolemici $(n=190)$, dove livelli più alti di cortisolo salivare erano associati a valori pressori significativamente superiori $[1,20]$. A ulteriore supporto dell'ipotesi che lega $11 \beta$-HSD1 e rischio cardiovascolare DM-associato vi sono i dati relativi all'utilizzo del Carbenoxolone, inibitore non selettivo degli enzimi $11 \beta$-HSD1 e 2 [21], nonché i risultati delle sperimentazioni di inibitori selettivi dell'enzima
$11 \beta$-HSD1 (INCB13739, RO-151, RO-838, P2202) [22]: in particolare, INCB13739, in un trial randomizzato controllato in doppio cieco condotto su 302 pazienti con DM 2 scarsamente controllato dalla monoterapia con metformina, ha mostrato miglioramenti significativi di emoglobina glicata, HOMA index e peso corporeo rispetto al placebo [22].

L'FH potrebbe sostenere il danno cognitivo associato al DM con un duplice effetto sull'ippocampo: alla desensitizzazione recettoriale che alimenta la secrezione di CRH ipotalamico si aggiunge, infatti, il danno ippocampale direttamente indotto dall'iperglicemia e alimentato dall'azione psicoattiva del cortisolo, che condiziona l'assunzione di cibo di bassa qualità, ma palatabile e ricco in calorie, zuccheri e grassi [1]. Ne risulta un maggior declino cognitivo generale, nei test di memoria operativa e velocità di calcolo (dati su 919 pazienti anziani con DM2 [23]), con evidenza di miglioramento della memoria verbale dopo 6 settimane di assunzione di Carbenoxolone [24].

\section{Sindrome dell'ovaio policistico (PCOS)}

Anovularietà, oligo-/amenorrea, irsutismo, acne, DM e insulino-resistenza, sovrappeso/obesità e ipertensione arteriosa sono manifestazioni comuni sia alla PCOS che alla SC $[2,3]$ : è pertanto ragionevole ricercare in donne con PCOS (6,6\% delle donne in età fertile) una CS, nonostante quest'ultima condizione sia estremamente più rara [2].

La diagnosi differenziale può essere indaginosa in quanto circa il 50\% delle donne con PCOS manifesta livelli di cortisolo libero urinario moderatamente elevati e i livelli di CBG sono spesso ridotti [2]. Inoltre, l'attivazione dell'asse HPA nella PCOS può essere secondaria all'aumentata attività di $5 \alpha$ - e $5 \beta$-reduttasi e dalla ridotta attività della $11 \beta$-HDS 1 adiposa [1]. È infine possibile che un certo grado di FH intervenga nella patogenesi della PCOS, inibendo il rilascio GnRH-mediato delle gonadotropine[1]: ciò è testimoniato dalla maggiore latenza tra i picchi di FSH/LH nelle donne con PCOS e livelli maggiori di cortisolo plasmatico [25].

Sebbene manchino studi estensivi sulle conseguenze dell'FH nella PCOS, alcuni studi suggeriscono che il cortisolo intervenga nella genesi di iperglicemia e DM perturbando il normale feedback glucosio-insulina a favore di ampi picchi insulinici e regolando negativamente gli eventi postrecettoriali indotti dall'insulina, quali uptake tissutale di glucosio, attività di piruvato deidrogenasi e glicogenosintasi [1].

\section{Sindrome delle apnee ostruttive notturne (OSAS)}

La fluttuazione notturna del cortisolo è correlata in modo complesso al sonno [26]: un'alterazione dell' asse HPA 
è stata pertanto associata ai disturbi del sonno, in primis l'OSAS, dove i frequenti risvegli notturni, la deprivazione di sonno, l'ipossia dovuta agli episodi di apnea e l'attività autonomica durante il sonno costituiscono potenti stimoli per la secrezione di cortisolo [1, 26]. Tuttavia, i dati esistenti in letteratura sono contrastanti (asse HPA preservato nell'OSAS come nei controlli, ridotta responsività dell'asse HPA con cortisolo inferiore nei pazienti OSAS rispetto ai controlli, FH nell'OSAS con ripristino dell'asse HPA parallelo al miglioramento dell'indice apnea-ipopnea dopo ossigenoterapia notturna) [26].

Anche nell'OSAS l'FH, in particolare il sovvertimento del ritmo circadiano del cortisolo a favore di un rialzo prevalentemente notturno, può contribuire all' aumento del rischio CV (specialmente stroke), in virtù della sua associazione con indici di insulino-resistenza e DM, obesità, ipertensione arteriosa, MS, insorgenza di aritmie e rilascio di citochine infiammatorie $[1,26]$.

\section{Lavoro a turni}

Nei lavoratori turnisti vi è un sovvertimento nel ritmo circadiano del cortisolo che, anche in assenza di malattie, li predispone a DM, obesità (evidenze isolate mostrano una correlazione diretta tra livelli di cortisolo nei capelli e BMI) e rischio CV [1]. Inoltre, uno studio condotto su quasi 500 lavoratori rumeni ha evidenziato come la rotazione su turni diversi si associ tipicamente a problemi sonno-relati (sonnolenza, ridotta qualità e durata del sonno) e posto l'attenzione sul possibile ruolo, causale o consequenziale, dell'FH nei quadri di obesità, ipertensione arteriosa e disturbo da attacchi di panico che tipicamente si riscontravano nei turnisti delle primissime ore del mattino [27].

\section{Conclusioni}

Nonostante le evidenze scientifiche non siano sempre concordi, si può con ragionevolezza ipotizzare che l'FH giochi un ruolo fondamentale nella genesi delle complicanze tipiche delle diverse entità dello pseudoCushing, anche in quelle condizioni (AN, BN, MD) in cui esso rappresenta un meccanismo adattativo allo stress. È pertanto fondamentale conoscerlo e riconoscerlo, così da poterne monitorare gli effetti nella pratica clinica e, appena necessario, instaurare un trattamento aggressivo con l'obiettivo primario di ottenerne la guarigione o, quantomeno, il controllo. Infine, laddove le alterazioni cliniche e biochimiche dell'FH non regrediscano nonostante terapia massimale, andrebbe riconsiderata la possibilità di una CS: il paziente dovrebbe essere quindi valutato $e$, in caso di dubbio diagnostico persistente, posto sotto stretto follow-up finalizzato a captare quanto più precocemente possibile l'evoluzione verso una vera CS.
Funding Note Open access funding provided by Università Politecnica delle Marche within the CRUI-CARE Agreement.

Conflitto di interesse Gli autori Marianna Martino e Giorgio Arnaldi dichiarano di non avere conflitti di interesse.

Consenso informato Lo studio presentato in questo articolo non ha richiesto sperimentazione umana.

Studi sugli animali Gli autori non hanno eseguito studi sugli animali.

Nota della casa editrice Springer Nature rimane neutrale in riguardo alle rivendicazioni giurisdizionali nelle mappe pubblicate e nelle affiliazioni istituzionali.

Open Access This article is licensed under a Creative Commons Attribution 4.0 International License, which permits use, sharing, adaptation, distribution and reproduction in any medium or format, as long as you give appropriate credit to the original author(s) and the source, provide a link to the Creative Commons licence, and indicate if changes were made. The images or other third party material in this article are included in the article's Creative Commons licence, unless indicated otherwise in a credit line to the material. If material is not included in the article's Creative Commons licence and your intended use is not permitted by statutory regulation or exceeds the permitted use, you will need to obtain permission directly from the copyright holder. To view a copy of this licence, visit http://creativecommons.org/licenses/by/4.0/.

\section{Bibliografia}

1. Tirabassi G, Boscaro M, Arnaldi G (2014) Harmful effects of functional hypercortisolism: a working hypothesis. Endocrine 46(3):370-386

2. Scaroni C, Albiger NM, Palmieri S et al (2020) Approach to patients with pseudo-Cushing's states. Endocr Connect 9(1):R1-R13

3. Arnaldi G, Mancini T, Tirabassi G et al (2012) Advances in the epidemiology, pathogenesis, and management of Cushing's syndrome complications. J Endocrinol Invest 35:434-448

4. Thakore JH, Richards PJ, Reznek RH et al (1997) Increased intra-abdominal fat deposition in patients with major depressive illness as measured by computed tomography. Biol Psychiatry 41:1140-1142

5. Broadley AJ, Korszun A, Abdelaal E et al (2006) Metyrapone improves endothelial dysfunction in patients with treated depression. J Am Coll Cardiol 48:170-175

6. Thomson F, Craighead M (2008) Inovative approaches for the treatment of depression: targeting the HPA axis. Neurochem Res 33:691-707

7. Michelson D, Stratakis C, Hill L et al (1996) Bone mineral density in women with depression. N Engl J Med 335:1176-1181

8. Steiger A, von Bardeleben U, Wiedemann K et al (1991) Sleep EEG and nocturnal secretion of testosterone and cortisol in patients with major endogenous depression during acute phase and after remission. J Psychiatr Res 25:169-177

9. Nasser FJ, Merula de Almeida M, Saraiva da Silva L et al (2016) Psychiatric disorders and cardiovascular system: heart-brain interaction. Int J Cardiovasc Sci 29(1):65-75

10. Castro-Fornieles J, Bargalló N, Lázaro L et al (2009) A crosssectional and follow-up voxel-based morphometric MRI study in adolescent anorexia nervosa. J Psychiatr Res 43:331-340 
11. Misra M, Prabhakaran R, Miller KK et al (2008) Prognostic indicators of changes in bone density measures in adolescent girls with anorexia nervosa-II. J Clin Endocrinol Metab 93:1292-1297

12. Naessén S, Carlström K, Glant R et al (2006) Bone mineral density in bulimic women-influence of endocrine factors and previous anorexia. Eur J Endocrinol 155:245-251

13. Abdelhadi Z, Bladbjerg EM, Møller Jensen D et al (2020) Venous thromboembolism in anorexia nervosa: four cases from a specialized unit. Indication for thromboprophylaxis? Eat Weight Disord 25(6): 1833-1837

14. Donoghue K, Rose A, Coulton S et al (2020) Doubleblind, placebo-controlled trial of mifepristone on cognition and depression in alcohol dependence. Trials 21(1):796

15. Van der Valk ES, Savas M, van Rossum EF (2018) Stress and obesity: are there more susceptible individuals? Curr Obes Rep 7(2):193-203

16. Stalder T, Steudte-Schmiedgen S, Alexander N et al (2017) Stress-related and basic determinants of hair cortisol in humans: a meta-analysis. Psychoneuroendocrinology 77:261-274

17. Noppe G, Van Den Akker ELT, De Rijke YB et al (2016) Long-term glucocorticoid concentrations as a risk factor for childhood obesity and adverse body-fat distribution. Int $\mathbf{J}$ Obes 40(10):1503-1509

18. Min L (2016) Functional hypercortisolism, visceral obesity, and metabolic syndrome. Endocr Pract 22(4):506-508

19. Gant CM, Minovic I, Binnenmars H et al (2018) Lower renal function is associated with derangement of 11-beta hydroxysteroid dehydrogenase in type 2 diabetes. J Endocr Soc 2(7):609-620
20. Oltmanns KM, Dodt B, Schultes B et al (2006) Cortisol correlates with metabolic disturbances in a population study of type 2 diabetic patients. Eur J Endocrinol 154:325-331

21. Andrews RC, Rooyackers O, Walker BR (2003) Effects of the 11 beta-hydroxysteroid dehydrogenase inhibitor carbenoxolone on insulin sensitivity in men with type 2 diabetes. J Clin Endocrinol Metab 88:285-291

22. Gregory S, Hill D, Grey B et al (2020) $11 \beta$-hydroxysteroid dehydrogenase type 1 inhibitor use in human disease - a systematic review and narrative synthesis. Metabolism 108:154246

23. Reynolds RM, Strachan MW, Labad J et al (2010) Morning cortisol levels and cognitive abilities in people with type 2 diabetes: the Edinburgh type 2 diabetes study. Diabetes Care 33:714-720

24. Sandeep TC, Yau JL, MacLullich AM et al (2004) 11Betahydroxysteroid dehydrogenase inhibition improves cognitive function in healthy elderly men and type 2 diabetics. Proc Natl Acad Sci USA 101:6734-6739

25. Zarković MJ, Penezić Z, Trbojević B et al (2001) Temporal coupling of luteinizing hormone and follicle stimulating hormone secretion in polycystic ovary syndrome. Gynecol Endocrinol 15:381-388

26. Bozic J, Galic T, Supe-Domic D et al (2016) Morning cortisol levels and glucose metabolism parameters in moderate and severe obstructive sleep apnea patients. Endocrine 53(3):730-739

27. Voinescu BI (2018) Common sleep, psychiatric, and somatic problems according to work schedule: an internet survey in an eastern European country. Int J Behav Med 25(4):456-464 